\title{
Schmidt decomposable products of projections
}

\author{
Esteban Andruchow and Gustavo Corach
}

June 19, 2017

\begin{abstract}
We characterize operators $T=P Q(P, Q$ orthogonal projections in a Hilbert space $\mathcal{H})$ which have a singular value decomposition. A spatial characterizations is given: this condition occurs if and only if there exist orthonormal bases $\left\{\psi_{n}\right\}$ of $R(P)$ and $\left\{\xi_{n}\right\}$ of $R(Q)$ such that $\left\langle\xi_{n}, \psi_{m}\right\rangle=0$ if $n \neq m$. Also it is shown that this is equivalent to $A=P-Q$ being diagonalizable. Several examples are studied, relating Toeplitz, Hankel and Wiener-Hopf operators to this condition. We also examine the relationship with the differential geometry of the Grassmann manifold of underlying the Hilbert space: if $T=P Q$ has a singular value decomposition, then the generic parts of $P$ and $Q$ are joined by a minimal geodesic with diagonalizable exponent.
\end{abstract}

2010 MSC: 47A05, 47A68, 47B35, 47B75.

Keywords: Projections, products of projections, differences of projections.

\section{Introduction}

Let $\mathcal{H}$ be a Hilbert space, $\mathcal{B}(\mathcal{H})$ the space of bounded linear operators, $\mathcal{P}(\mathcal{H}) \subset \mathcal{B}(\mathcal{H})$ the set of orthogonal projections. In what follows $R(T)$ denotes the the range of $T \in \mathcal{B}(\mathcal{H})$ and $N(T)$ its nullspace. Given a closed subspace $\mathcal{S} \subset \mathcal{H}$, the orthogonal projection onto $\mathcal{S}$ is denoted by $P_{\mathcal{S}}$. In this paper we study part of the set $\mathcal{P} \cdot \mathcal{P}=\{P Q: P, Q \in \mathcal{P}(\mathcal{H})\}$, namely, the subset of all $T=P Q$ such that $T^{*} T=P Q P$ is diagonalizable. Operators in $\mathcal{P} \cdot \mathcal{P}$ are special cases of generalized Toeplitz operators as well as of Wiener-Hopf operators. As we shall see in a section of examples, they give rise to classical Toeplitz and Wiener-Hopf operators. Therefore this paper can be regarded as the study of operators in these classes, having a diagonal structure.

Also this paper is a kind of sequel to [8], [3] and [4], the first concerned with the whole set $\mathcal{P} \cdot \mathcal{P}$, the other two with $\mathcal{P} \cdot \mathcal{P} \cap \mathcal{K}(\mathcal{H})$, where $\mathcal{K}(\mathcal{H})$ denotes the ideal of compact operators acting in $\mathcal{H}$. Compact operators $T$ satisfy that $T^{*} T$ is diagonalizable.

We shall say that $T$ is S-decomposable if it has a singular value (or Schmidt) decomposition $[24]$,

$$
T=\sum_{n \geq 1} s_{n}\left\langle\quad, \xi_{n}\right\rangle \psi_{n}=\sum_{n \geq 1} s_{n} \psi_{n} \otimes \xi_{n},
$$

where $\left\{\xi_{n}: n \geq 1\right\}$ and $\left\{\psi_{n}: n \geq 1\right\}$ are orthonormal systems, and $s_{n}>0$. In this case, $\left\{\psi_{n}\right\}$, $\left\{x i_{n}\right\}$ are orthonormal bases of $\overline{R(T)}, N(T)^{\perp}$, respectively and $T \xi_{n}=s_{n} \psi_{n}, T^{*} \psi_{n}=s_{n} \xi_{n}$, $T^{*} T \xi_{n}=s_{n}^{2} \xi_{n}, T T^{*} \psi_{n}=s_{n}^{2} \psi_{n}$ for all $n \geq 1$. 
Clearly, $T$ is S-decomposable if and only if $T^{*} T$ (equivalently $T T^{*}$ ) is diagonalizable, if and only if $T^{*}$ is S-decomposable. Also it is clear that if $U, V$ are unitary operators, $T$ is S-decomposable if and only if $U T V$ is S-decomposable.

This paper is devoted to the study of the operators $T \in \mathcal{P} \cdot \mathcal{P}$ which are S-decomposable.

Let us describe the contents of the paper. In Section 2 we prove that $T=P Q$ is $\mathrm{S}$ decomposable if and only if there exist orthonormal bases $\left\{\xi_{n}\right\}$ of $R(P)$ and $\left\{\psi_{n}\right\}$ of $R(Q)$ such that $\left\langle\xi_{n}, \psi_{m}\right\rangle \neq 0$ if $n \neq m$. We also prove that $T=P Q$ is S-decomposable if and only if $A=P-Q$ is diagonalizable. This result is based on a Theorem by Chandler Davis ([10], Theorem 6.1), which characterizes operators which are the difference of two projections. A recent treatment of these operators can be found in [2]. The S-decomposability of $P Q$ is equivalent to that of $P(1-Q),(1-P) Q$ and $(1-P)(1-Q)$. As a corollary we prove that $P-Q$ is diagonalizable if and only if $P+Q$ is, the eigenvalues $\pm \lambda_{n}$ of $P-Q$ which are different from 0,1 correspond with the eigenvalues $1 \pm\left(1-\lambda_{n}\right)^{2}$, with the same multiplicity. Section 3 contains several interesting classes of examples of S-decomposable operators in $\mathcal{P}$. $\mathcal{P}$. If $\mathcal{H}=L^{2}\left(\mathbb{R}^{n}\right)$ and $I, J \subset \mathbb{R}^{n}$ are Lebesgue measurable set with finite positive measure, define $P_{I} f=\chi_{I} f$ and $Q_{J} f=\left(P_{J} \hat{f}\right)$, for $f \in \mathcal{H}$. Here $\chi_{A}$ denotes the characteristic function of $A \subset \mathbb{R}^{n}$ and ${ }^{\wedge}$, denote the Fourier-Plancherel transform and its inverse. The product $P_{I} Q_{J}$ is a proper Wiener-Hopf operator, is also known as a concentration operator, and its study is related to mathematical formulations of the Heisenberg uncertainty principle. The reader is referred to [25], [19], [11], [12] for results concerning these products. Under the conditions described above, $P_{I} Q_{J}$ is a HilbertSchmidt operator, thus S-decomposable. This implies that also $P_{I^{\prime}} Q_{J^{\prime}}$ is $\mathrm{S}$-decomposable (but non compact) when $I^{\prime}$ or $J^{\prime}$ have co-finite measure. It should be mentioned that the spectral description of the $P_{I} Q_{J}$ is no easy task (see [25] for the case when $I, J$ are intervals in $\mathbb{R}$ ). Another interesting family of examples is obtained if $\mathcal{H}=L^{2}(\mathbb{T})$ and $\mathcal{H}$ is decomposed as $\mathcal{H}=\mathcal{H}_{+} \oplus \mathcal{H}_{-}$, where $\mathcal{H}_{+}=H^{2}(\mathbb{T})$. If $\varphi, \psi$ are continuous functions with modulus one, put $P=1-P_{\varphi \mathcal{H}_{+}}$and $Q=P_{\psi \mathcal{H}_{+}}$. Then $P Q$ is a unitary operator times a Hankel operator with continuous symbol, and therefore a compact operator by a Theorem by Hartman [16]. Then $(1-P) Q$ is a unitary operator times a Toeplitz operator, and a non compact S-decomposable operator. On the other hand, using a result by Howland ([18], Theorem 9.2), one can find convenient non-continuous $\varphi, \psi$ such that $P Q$ is not $\mathrm{S}$-decomposable.

In Section 4 we prove that, for two closed subspaces $\mathcal{S}, \mathcal{T}$ of $\mathcal{H}$, the operator $T=P_{\mathcal{S}} P_{\mathcal{T}}$ is S-decomposable if and only if there exist isometries $X, Y: \ell^{2} \rightarrow \mathcal{H}$ with $R(X)=\mathcal{S}, R(Y)=\mathcal{T}$ such that $X^{*} Y \in \mathcal{B}\left(\ell^{2}\right)$ is a diagonal matrix.

In Section 5 we characterize S-decomposability in terms of what we call Davis' symmetry $V$ : given two projections $P, Q$, the decomposition $\mathcal{H}=N(P+Q-1) \oplus N(P+Q-1)^{\perp}$ reduces simultaneously $P$ and $Q$. They act non trivially on the second subspace $\mathcal{H}^{\prime}=N(P+Q-1)^{\perp}$. Denote by $P^{\prime}$ and $Q^{\prime}$ the restrictions of $P$ and $Q$ to this subspace. Then the isometric part in the polar decompostion of $P^{\prime}+Q^{\prime}-1$ is a selfadjoint unitary operator $V$ which satisfies $V P^{\prime} V=Q^{\prime}, V Q^{\prime} V=P^{\prime}$. We relate these operator with the differential geometry of the space $\mathcal{P}\left(\mathcal{H}^{\prime}\right)$ of projections in $\mathcal{H}^{\prime}$ (or Grassmann manifold of $\mathcal{H}^{\prime}$ ). Specifically, with the unique short geodesic curve joining $P^{\prime}$ and $Q^{\prime}$ in $\mathcal{P}\left(\mathcal{H}^{\prime}\right)$. For instance, it is shown that $P Q$ is S-decomposable if and only if the velocity operator of the unique geodesic joining $P^{\prime}$ and $Q^{\prime}$ is diagonalizable.

In Section 5 it is shown that any contraction $\Gamma \in \mathcal{B}(\mathcal{H})$ is the 1,1 entry of a unitary operator times a product of projections acting in $\mathcal{H} \times \mathcal{H}$. 


\section{Products and differences of projections}

If $T \in \mathcal{P} \cdot \mathcal{P}$, then $T=P_{\overline{R(T)}} P_{N(T)^{\perp}}$. This is a result of T. Crimmins (unpublished; there is a proof in [23] Theorem 8). Moreover, Crimmins proved that $T \in \mathcal{B}(\mathcal{H})$ belongs to $\mathcal{P} \cdot \mathcal{P}$ if and only if $T T^{*} T=T^{2}$ [23]. However, the factorization $T=P_{\overline{R(T)}} P_{N(T)^{\perp}}$ is one among among many others. In [8], Theorem 3.7, it is proved that if $T \in \mathcal{P} \cdot \mathcal{P}$, then $T=P_{\mathcal{S}} P_{\mathcal{T}}$ if and only if

$$
\overline{R(T)} \subset \mathcal{S}, N(T)^{\perp} \subset \mathcal{T} \text { and }(\mathcal{S} \ominus \overline{R(T)}) \oplus\left(\mathcal{T} \ominus N(T)^{\perp}\right) \subset R(T)^{\perp} \cap N(T) .
$$

In [8], for any $T \in \mathcal{P} \cdot \mathcal{P}$ the set of all pairs $(\mathcal{S}, \mathcal{T})$ of closed subspaces such that $T=P_{\mathcal{S}} P_{\mathcal{T}}$ is denoted by $\mathcal{X}_{T}$. Our first result is a characterization of $\mathcal{X}_{T}$ for $\mathrm{S}$-decomposable $T$. The proof is essentially that of Theorem 4.1 in [4], where $T$ is supposed to be a compact element of $\mathcal{P} \cdot \mathcal{P}$. We include a proof for the reader's convenience.

Theorem 2.1. Let $\mathcal{S}, \mathcal{T} \subset \mathcal{H}$ be closed subspaces of $\mathcal{H}$. Then $T=P_{\mathcal{S}} P_{\mathcal{T}}$ is $S$-decomposable if and only if there exist orthonormal bases $\left\{\psi_{n}: n \geq 1\right\}$ of $\mathcal{S},\left\{\xi_{n}: n \geq 1\right\}$ of $\mathcal{T}$ such that $\left\langle\xi_{n}, \psi_{m}\right\rangle=0$ if $n \neq m$. In such case, the numbers $\left|\left\langle\xi_{n}, \psi_{n}\right\rangle\right|$ are the singular values of $T$.

Proof. Suppose that $\left\{\psi_{n}\right\},\left\{\xi_{n}\right\}$ are orthonormal bases of $\mathcal{S}, \mathcal{T}$ respectively, such that

$$
\left\langle\psi_{n}, \xi_{m}\right\rangle=0 \text { for } n \neq m \text {. }
$$

Therefore

$$
P_{\mathcal{S}} P_{\mathcal{T}}=\left(\sum_{n \geq 1}\left\langle, \psi_{n}\right\rangle \psi_{n}\right)\left(\sum_{m \geq 1}\left\langle\quad, \xi_{m}\right\rangle \xi_{m}\right)=\sum_{n \geq 1}\left\langle\psi_{n}, \xi_{n}\right\rangle \psi_{n} \otimes \xi_{n} .
$$

In order to get the Schmidt decomposition of $P_{\mathcal{S}} P_{\mathcal{T}}$, we only need to replace $\left\langle\psi_{n}, \xi_{n}\right\rangle$ by the appropriate sequence of positive numbers: write $\left\langle\psi_{n}, \xi_{n}\right\rangle=e^{i \theta_{n}}\left|\left\langle\psi_{n}, \xi_{n}\right\rangle\right|$ and replace $\psi_{n}$ by $\psi_{n}^{\prime}=e^{-i \theta_{n}} \psi_{n}$. Then $\left\{\psi_{n}^{\prime}\right\}$ is still an orthonormal basis of $\mathcal{S}$, and

$$
\left\langle\psi_{n}^{\prime}, \xi_{n}\right\rangle=\left|\left\langle\psi_{n}, \xi_{n}\right\rangle\right|=s_{n}
$$

are the singular values in the decomposition

$$
P_{\mathcal{S}} P_{\mathcal{T}}=\sum_{n \geq 1}\left|\left\langle\psi_{n}, \xi_{n}\right\rangle\right| \psi_{n}^{\prime} \otimes \xi_{n}
$$

This shows that $P_{\mathcal{S}} P_{\mathcal{T}}$ is S-decomposable.

Conversely, if $T=P_{\mathcal{S}} P_{\mathcal{T}}$ is S-decomposable it has a singular value decomposition

$$
T=\sum_{n \geq 1} s_{n} \psi_{n} \otimes \xi_{n}
$$

and it holds that $T^{2}=T T^{*} T$. Then

$$
T^{*}=\sum_{n \geq 1} s_{n}\left\langle, \psi_{n}\right\rangle \xi_{n}, T T^{*} T=\sum_{n \geq 1} s_{n}^{3}\left\langle, \xi_{n}\right\rangle \psi_{n},
$$

and

$$
T^{2}=\sum_{n, m \geq 1} s_{n} s_{m}\left\langle\psi_{n}, \xi_{n}\right\rangle\left\langle, \xi_{n}\right\rangle \psi_{n}=\sum_{n \geq 1} s_{n}\left(\sum_{m \geq 1}\left\langle, s_{m}\left\langle\xi_{n}, \psi_{m}\right\rangle \xi_{m}\right) \psi_{n}\right.
$$


Using $T T^{*} T=T^{2}$ we get, for each $n \geq 1$

$$
\sum_{m \geq 1} s_{n} s_{m}\left\langle\xi_{n}, \psi_{m}\right\rangle \xi_{m}=s_{n}^{3} \xi_{n}
$$

Then $\left\langle\xi_{n}, \psi_{m}\right\rangle=0$ if $n \neq m$ and $s_{n}=\left\langle\xi_{n}, \psi_{n}\right\rangle$. Finally, we can extend the orthonormal bases $\left\{\psi_{n}\right\}$ of $\overline{R(T)}$ and $\left\{\xi_{n}\right\}$ of $N(T)^{\perp}$ to orthonormal bases of $\mathcal{S}$ and $\mathcal{T}$. In fact, if $\psi \in \mathcal{S} \ominus \overline{R(T)}$ and $\xi \in \mathcal{T} \ominus N(T)^{\perp}$, then

$$
\langle\psi, \xi\rangle=0
$$

because

$$
(\mathcal{S} \ominus \overline{R(T)}) \oplus\left(\mathcal{T} \ominus N(T)^{\perp}\right) \subset R(T)^{\perp} \cap N(T) .
$$

Next, we show that $T=P Q$ is S-decomposable if and only if $A=P-Q$ is diagonalizable, and establish the relation between the singular values of $T$ and the eigenvalues of $A$. We present this equivalence as two separate theorems, to avoid too long a statement.

Theorem 2.2. Suppose that $T=P Q$ is $S$-decomposable with singular values $s_{n}$. Then $A=$ $P-Q$ is diagonalizable, with eigenvalues $\pm\left(1-s_{n}^{2}\right)^{1 / 2}, n \geq 1$, plus, eventually, $0,-1$ and 1 .

Proof. Put as above $T=\sum_{n \geq 1} s_{n} \psi_{n} \otimes \xi_{n}$, with $\xi_{n} \in R(Q)$ and $\psi_{n} \in R(P)$. First note that $s_{n} \leq s_{1}=\|T\| \leq\|P\|\|Q\| \leq 1$. Moreover, $s_{1}=1$ means that $T \xi_{1}=\eta_{1}$ and thus $\left\|P\left(Q \xi_{1}\right)\right\|=$ $1=\left\|\xi_{1}\right\| \geq\left\|Q \xi_{1}\right\| \geq\left\|P\left(Q \xi_{1}\right)\right\|$, i.e., $\xi_{1} \in R(Q)$ and $Q \xi_{1}=\xi_{1} \in R(P)$. Then $\xi_{1}=\psi_{1}$. The same happens for all $n$ such that $s_{n}=1$ : the associated vectors $\xi_{n}=\psi_{n}$ generate $R(P) \cap R(Q)$. Note that $A=P-Q$ is trivial in this subspace.

Suppose that $s_{k}<1$. Apparently,

$$
A \xi_{k}=P \xi_{k}-Q \xi_{k}=P Q \xi_{k}-\xi_{k}=T \xi_{k}-\xi_{k}=s_{k} \psi_{k}-\xi_{k}
$$

and

$$
A \psi_{k}=P \psi_{k}-Q \psi_{k}=P \psi_{k}-Q P \psi_{k}=\psi_{k}-T^{*} \psi_{k}=\psi_{k}-s_{k} \xi_{k}
$$

Then

$$
A^{2} \xi_{k}=\left(1-s_{k}^{2}\right) \xi_{k} . \quad A^{2} \psi_{k}=\left(1-s_{k}\right)^{2} \psi_{k} .
$$

Since $s_{k}=\left|\left\langle\xi_{k}, \psi_{k}\right\rangle\right|<1$ and $\left\|\xi_{k}\right\|=\left\|\psi_{k}\right\|=1$, it follows that $\xi_{k}, \psi_{k}$ span a two-dimensional eigenspace for $A^{2}$, with eigenvalue $1-s_{k}^{2}$. Then

$$
\nu_{k}=\left(\left(1-s_{k}^{2}\right)^{1 / 2}-1\right) \xi_{k}+s_{k} \psi_{k} \text { and } \omega_{k}=\left(-\left(1-s_{k}^{2}\right)^{1 / 2}-1\right) \xi_{k}+s_{k} \psi_{k}
$$

are orthogonal eigenvectors for $A$, with eigenvalues $\left(1-s_{k}^{2}\right)^{1 / 2}$ and $-\left(1-s_{k}^{2}\right)^{1 / 2}$, respectively.

The orthogonal systems $\xi_{k}$ and $\psi_{k}$ can be extended to orthonormal bases of $R(P)$ and $R(Q)$, respectively (as in the proof of Theorem 2.1). On the extension of the system $\xi_{k}$, i.e., $R(P) \ominus R(T), A=P-Q$ equals 1 . On the extension of $\psi_{k}, R(Q) \ominus N(T)^{\perp}, A$ equals -1 . Together, these extended systems span $R(P)+R(Q)$, and here $A$ is diagonalizable. On the orthogonal complement of this subspace, namely $N(P)^{\perp} \cap N(Q)^{\perp}, A$ is trivial. 
Remark 2.3. Note that, except for 1 and -1 , the eigenvalues $\left(1-s_{k}^{2}\right)^{1 / 2}$ and $-\left(1-s_{k}^{2}\right)^{1 / 2}$ of $A$ have the same multiplicity. Also note that

$$
N(A-1)=R(P) \cap N(Q), \quad N(A+1)=N(P) \cap R(Q),
$$

and $N(A)=R(P) \cap R(Q) \oplus N(P) \cap N(Q)$.

The above result has a converse. In [10] Chandler Davis proved that operators $A=P-Q$ are characterized as follows: in the generic part of $A$, namely

$$
\mathcal{H}_{0}=\{N(A) \oplus N(A-1) \oplus N(A+1)\}^{\perp},
$$

which reduces $P, Q$ and $A$, if we denote $P_{0}=\left.P\right|_{\mathcal{H}_{0}}, Q_{0}=\left.Q\right|_{\mathcal{H}_{0}}$ and

$$
A_{0}=\left.A\right|_{\mathcal{H}_{0}}=P_{0}-Q_{0}
$$

there exists a symmetry $V\left(V^{*}=V^{-1}=V\right)$ such that $V A=-A V$ and

$$
P_{0}=P_{V}=\frac{1}{2}\left\{1+A_{0}+V\left(1-A_{0}^{2}\right)^{1 / 2}\right\} \quad, \quad Q_{0}=Q_{V}=\frac{1}{2}\left\{1-A_{0}+V\left(1-A_{0}^{2}\right)^{1 / 2}\right\} .
$$

$V$ is characterized by these properties. With these notations we have:

Theorem 2.4. If $A=P-Q$ is diagonalizable with (non nil) eigenvalues $\pm \lambda_{n}\left(0<\left|\lambda_{n}\right|<1\right.$ ) and \pm 1 , then $T=P Q$ is $S$-decomposable with singular values $\left(1-\lambda_{n}^{2}\right)^{1 / 2}$ and 1 .

Proof. On the non generic parts $N(A-1) \oplus N(A+1)$, T equals zero. In $N(A)=R(P) \cap R(Q) \oplus$ $N(P) \cap N(Q), T$ is

$$
1 \oplus 0 .
$$

Thus $P Q$ is diagonal (thus $\mathrm{S}$-decomposable) in $\mathcal{H}_{0}^{\perp}$. In $\mathcal{H}_{0}$, after straightforward computations (note that $V$ commutes with $A_{0}^{2}$ ) one has

$$
P_{0} Q_{0}=P_{V} Q_{V}=\frac{V}{2}\left\{V\left(1-A_{0}^{2}\right)^{1 / 2}+1-A_{0}\right\}\left(1-A_{0}^{2}\right)^{1 / 2}
$$

Since $A_{0}$ is diagonalizable, and there exists the symmetry $V$ associated to $P_{0}$ and $Q_{0}$, which intertwines $A_{0}$ with $-A_{0}$, it follows that $A_{0}$ is of the form

$$
A_{0}=\sum_{n \geq 1} \lambda_{n}\left(E_{n}-F_{n}\right)
$$

where $E_{n}, F_{n}(n \geq 1)$ are pairwise orthogonal projections with $\operatorname{dim} R\left(E_{n}\right)=\operatorname{dim} R\left(F_{n}\right)=$ $m_{n} \leq \infty$. The eigenvalues $\lambda_{n}$ of $A_{0}$ are different from \pm 1 , because $N\left(A_{0} \pm 1\right)=\{0\}$. Fix an orthonormal basis $\left\{\nu_{k}^{n}: 1 \leq k \leq m_{n}\right\}$ for $R\left(E_{n}\right)$. The fact that $V A=-A V$ implies that $V$ maps (the $\lambda_{n}$-eigenspace) $R\left(E_{n}\right)$ onto (the $-\lambda_{n}$-eigenspace) $R\left(F_{n}\right)$, and back. Then we can consider for $R\left(F_{n}\right)$ the orthonormal basis given by $\omega_{k}^{n}=V \nu_{k}^{n}$. Thus also $V \omega_{k}^{n}=\nu_{k}^{n}$. Then

$$
P_{0} Q_{0} \nu_{k}^{n}=\frac{1}{2}\left(1-\lambda_{n}^{2}\right) \nu_{k}^{n}+\frac{1}{2}\left(1-\lambda_{n}\right)\left(1-\lambda_{n}^{2}\right)^{1 / 2} \omega_{k}^{n}
$$

and

$$
P_{0} Q_{0} \omega_{k}^{n}=\frac{1}{2}\left(1-\lambda_{n}^{2}\right) \omega_{k}^{n}+\frac{1}{2}\left(1+\lambda_{n}\right)\left(1-\lambda_{n}^{2}\right)^{1 / 2} \nu_{k}^{n}
$$


It follows that the 2-dimensional subspace generated by (the orthonormal vectors) $\nu_{k}^{n}$ and $\omega_{k}^{n}$ is invariant for $P_{0} Q_{0}$. The matrix of $P_{0} Q_{0}$ restricted to this subspace (in this basis) is

$$
\frac{1}{2}\left(\begin{array}{cc}
\left(1-\lambda_{n}^{2}\right) & \left(1+\lambda_{n}\right)\left(1-\lambda_{n}^{2}\right)^{1 / 2} \\
\left(1-\lambda_{n}\right)\left(1-\lambda_{n}^{2}\right)^{1 / 2} & \left(1-\lambda_{n}^{2}\right)
\end{array}\right),
$$

whose singular values are 0 and $\left(1-\lambda_{n}^{2}\right)^{1 / 2}$. In the orthonormal basis $\left\{\nu_{k}^{n}, \omega_{k}^{n}\right\}$ of $\mathcal{H}_{0}$ (paired in this fashion), the operator $P_{0} Q_{0}$ is block-diagonal, with $2 \times 2$ blocks. It follows that $P Q$ is S-decomposable with singular values $\left(1-\lambda_{n}^{2}\right)^{1 / 2}$ and, eventually, 1 . The singular value 1 occurs only if $R(P) \cap R(Q) \neq\{0\}$.

Remark 2.5. The multiplicity of $\left(1-\lambda_{n}^{2}\right)^{1 / 2}$ as a singular value of $P Q$ is $m_{n}$.

Remark 2.6. From the above results, which relate eigenvalues of $P-Q$ and singular values of $P Q$, it follows that if $P Q$ is compact, and either $P$ or $Q$ have infinite rank, then $\|P-Q\|=1$. Indeed, if $P Q$ is compact, the singular values accumulate eventually at 0 , and therefore the eigenvalues of $A$ accumulate at 1 . However, this result holds with more generality. It is a simple exercise that if $p \neq q$ are non nil projections in a $\mathrm{C}^{*}$-algebra such that $p q=0$, then $\|p-q\|=1$. Our case consists in reasoning in the Calkin algebra: $p=\pi(P), q=\pi(Q)$, where $\pi: \mathcal{B}(\mathcal{H}) \rightarrow \mathcal{B}(\mathcal{H}) / \mathcal{K}(\mathcal{H})$ is the quotient homomorphism. Then

$$
1 \leq\|p-q\| \leq\|P-Q\| \leq 1
$$

The following result will be useful to provide further examples. In a special case (see Example 3.1 in Section 3), it was proven by M. Smith ([26], Th. 3.1)

Proposition 2.7. $P Q$ is $S$-decomposable if and only if $P(1-Q)$ is $S$-decomposable (and therefore if and only if $(1-P) Q$ or $(1-P)(1-Q)$ are $S$-decomposable $)$.

Proof. $P(1-Q)$ is S-decomposable if and only if $P(1-Q) P=P-P Q P$ is diagonalizable. This operator acts non trivially only in $R(P)$. Thus, it is diagonalizable if and only if it is diagonalizable in $R(P)$. Adding $1-P$ (equal to the identity in $N(P)$ ), one obtains that this latter fact is equivalent to $1-P Q P=1-P \oplus P-P Q P$ being diagonalizable in $\mathcal{H}=N(P) \oplus R(P)$. Clearly $1-P Q P$ is diagonalizable if and only if $P Q P$ also is, i.e., if and only if $P Q$ is $\mathrm{S}$ decomposable.

As a direct consequence of this fact, one obtains the following corollary

Corollary 2.8. Let $P, Q$ be projections. Then $P-Q$ is diagonalizable if and only if $P+Q$ is diagonalizable. In that case, $\lambda_{n}$ is an eigenvalue of $P-Q$ with $0<\left|\lambda_{n}\right|<1$ if and only if $1 \pm\left(1-\lambda_{n}\right)^{2}$ is an eigenvalue of $P+Q$, with the same multiplicity.

Proof. By the above results, any eigenvalue $\lambda_{n}= \pm\left(1-s_{n}^{2}\right)^{1 / 2}$, where $s_{n}$ is a singular value of $P Q$, or equivalently, $s_{n}^{2}$ is an eigenvalue of $P Q P$. On the other hand, from the proof of Proposition 2.7, the eigenvalues of

$$
1-P Q P=1-P \oplus P Q^{\perp} P
$$

are 1 , and $1-s_{n}^{2}$. Then again by Theorem 2.2 , the eigenvalues of $P-Q^{\perp}=P+Q-1$ are $\pm s_{n}$, and thus the eigenvalues of $P+Q$ are $1 \pm s_{n}=1 \pm\left(1-\lambda_{n}^{2}\right)^{1 / 2}$. Since $P-Q$ is a difference of 
projections, the eigenvalues $+\lambda$ and $-\lambda$ (when $0<|\lambda|<1$ ) have the same multiplicity (see [2]), and by the above results, these add up to the multiplicity of $s=\left(1-\lambda^{2}\right)^{1 / 2}$ as a singular value of $P Q$. This number clearly equals the multiplicity of $\left(1-s^{2}\right)^{1 / 2}$ as a singular value of $P Q^{\perp}$. Note that $P+Q-1=P-Q^{\perp}$ is also a difference of projections, therefore the multiplicities of $\pm s= \pm\left(1-\lambda^{2}\right)^{1 / 2}$ coincide $(0<s<1)$.

Remark 2.9. The multiplicity of 1 as an (eventual) eigenvalue of $P-Q$ is the dimension of $R(P) \cap N(Q)$, the multiplicity of -1 is the dimension of $N(P) \cap R(Q)$, the sum of these multiplicities is the multiplicity of 0 in $P-Q^{\perp}$, or the multiplicity of 1 in $P+Q$. Similarly, the multiplicity of 0 in $P-Q$ equals the sum of the multiplicities of 0 and 2 in $P+Q$.

Remark 2.10. To study the examples in the next section, it will also be useful to note that if $P$ has infinite rank and $P Q$ is compact, then $P(1-Q)$ is S-decomposable but non compact.

\section{Examples}

Example 3.1. Let $I, J \subset \mathbb{R}^{n}$ be Lebesgue-measurable sets of finite measure. Let $P_{I}, Q_{J}$ be the projections in $L^{2}\left(\mathbb{R}^{n}, d x\right)$ given by

$$
P_{I} f=\chi_{I} f \quad \text { and } \quad Q_{J} f=\left(\chi_{J} \hat{f}\right) \sim
$$

where $\chi_{L}$ denotes the characteristic function of the set $L$. Equivalently, denoting by $U_{\mathcal{F}}$ the Fourier transform regarded as a unitary operator acting in $L^{2}\left(\mathbb{R}^{n}, d x\right)$, then

$$
P_{I}=M_{\chi_{I}} \text { and } Q_{J}=U_{\mathcal{F}}^{*} M_{\chi_{J}} U_{\mathcal{F}}
$$

In [11] (Lemma 2) it is proven that $P_{I} Q_{J}$ is a Hilbert-Schmidt operator. See also [12]. Then $T=P_{I} Q_{J}$ is S-decomposable (with square summable singular values) These products play a relevant role in operator theoretic formulations of the uncertainty principle [11], [12].

In this case one has the spectral picture of $A=P_{I}-Q_{J}$. It is known [19], [12] that

$$
N\left(P_{I}\right) \cap N\left(Q_{J}\right)=R\left(P_{I}\right) \cap N\left(Q_{J}\right)=N\left(P_{I}\right) \cap R\left(Q_{J}\right)=\{0\}
$$

and $R\left(P_{I}\right) \cap R\left(Q_{J}\right)$ is infinite dimensional. Thus $N(A)=R\left(P_{I}\right) \cap R\left(Q_{J}\right)$ is infinite dimensional, $N(A \pm 1)=\{0\}$, and the eigenvalues of $A$ are of the form $\pm\left(1-s_{k}^{2}\right)^{1 / 2}$, where the sequence $s_{k}$ belongs to $\ell^{2}(\mathbb{Z})$. In special cases, e.g. $I=[0, T], J=[-\Omega, \Omega]$ intervals in $\mathbb{R}$, the eigenfunctions are known and the eigenvalues have multiplicity one [17].

If one relaxes the condition that the sets be of finite measure, $P_{I} Q_{J}$ ceases to be compact. Using Proposition 2.7, one obtains non compact examples: replacing the above conditions by $\left|\mathbb{R}^{n} \backslash I\right|<\infty$ or $\left|\mathbb{R}^{n} \backslash J\right|<\infty$ (see also [26], one obtains non-compact, S-decomposable products of projections.

Note also that, due to Theorem 2.1, in the above cases (i.e. both $I$ and $J$ have finite or cofinite measure), the subspaces $R\left(P_{I}\right)=\left\{f \in L^{2}\left(\mathbb{R}^{n}\right):\left.f\right|_{\mathbb{R}^{n} \backslash I}=0\right\}$ and $R\left(Q_{J}\right)=\left\{g \in L^{2}\left(\mathbb{R}^{n}\right)\right.$ : $\left.\left.\hat{g}\right|_{\mathbb{R}^{n} \backslash J}=0\right\}$ have orthonormal bases $\left\{f_{n}\right\}$ and $\left\{g_{n}\right\}$, repectively, which satisfy $\left\langle f_{n}, g_{m}\right\rangle=0$ if $n \neq m$.

We study more carefully the case

$$
I=[0,+\infty), \quad J=[-1,1],
$$


not covered above. Straightforward computations (see [19]) show that the operator $P_{I} Q_{J}$, acting in $L^{2}(0,+\infty)$ is given by

$$
P_{I} Q_{J} P_{I} f(x)=\frac{1}{\pi} \int_{0}^{\infty} \operatorname{sinc}(x-t) f(t) d t .
$$

Let us prove that $P_{I} Q_{J} P_{I}$ is non compact. For $n \in \mathbb{N}$, let

$$
e_{n}(x)=\left\{\begin{array}{l}
e^{-\frac{1}{n} x} e^{i x}, \text { if } x \geq 0 \\
0 \text { otherwise }
\end{array}\right.
$$

Apparently $e_{n} \in L^{2}(\mathbb{R})$ and $\left\|e_{n}\right\|_{2}^{2}=\frac{n}{2}$. Note that

$$
P_{I} Q_{J} P_{I} \frac{e_{n}(x)}{\left\|e_{n}\right\|_{2}}=\frac{\sqrt{2}}{\pi \sqrt{n}}\left\{\int_{0}^{x} \operatorname{sinc}(x-t) e_{n}(t) d t+\int_{x}^{\infty} \operatorname{sinc}(x-t) e_{n}(t) d t\right\} .
$$

Changing variables $v=x-t$ in the first integral and $u=t-x$ in the second, one obtains

$$
\frac{\sqrt{2}}{\pi \sqrt{n}}\left\{e_{n}(x) \int_{0}^{x} \operatorname{sinc}(v) e_{n}(-v) d v+e_{n}(x) \int_{0}^{\infty} \operatorname{sinc}(u) e_{n}(u) d u\right\}
$$

The second integral, which we shall denote $\lambda_{n}$, can be computed. Denote by $\mathbb{L}$ the usual Laplace transform. Then

$$
\begin{aligned}
\lambda_{n} & =\int_{0}^{\infty} \operatorname{sinc}(u) e_{n}(u) d u=\int_{0}^{\infty} \operatorname{sinc}(u) \cos (u) e^{-\frac{1}{n} u} d u+i \int_{0}^{\infty} \operatorname{sinc}(u) \sin (u) e^{-\frac{1}{n} u} d u \\
& =\left.\mathbb{L}\left(\frac{\sin (t)}{t} \cos (t)\right)\right|_{t=\frac{1}{n}}+\left.i \mathbb{L}\left(\frac{\sin ^{2}(t)}{t}\right)\right|_{t=\frac{1}{n}}=\frac{\pi}{2}-\arctan \left(\frac{1}{n}\right)+i \frac{1}{4} \ln \left(1+4 n^{2}\right) .
\end{aligned}
$$

Let us denote by $F_{n}(x)$ the left hand integral,

$$
F_{n}(x)=\int_{o}^{x} \operatorname{sinc}(t) e_{n}(-t) d t
$$

Lemma 3.2. With the current notations,

$$
\frac{1}{\left\|e_{n}\right\|_{2}}\left\|P_{I} Q_{J} P_{I} e_{n}-e_{n}\right\|_{2} \rightarrow 0, \text { as } n \rightarrow \infty
$$

Proof. Compute

$$
\left\langle e_{n} F_{n}, e_{n}\right\rangle=\int_{0}^{\infty} e_{n}(x) F_{n}(x) \bar{e}_{n}(x) d x=\int_{0}^{\infty} e^{-\frac{2}{n} x} F_{n}(x) d x .
$$

Integrating by parts, and using that (by means of the L'Hospital rule !), we get

$$
\lim _{x \rightarrow+\infty} \frac{F_{n}(x)}{e^{\frac{2}{n} x}}=\lim _{x \rightarrow \infty} \frac{n}{2} \frac{\sin c(x) e_{n}(-x)}{e^{\frac{2}{n} x}}=\frac{n}{2} \lim _{x \rightarrow+\infty} \frac{\operatorname{sinc}(x)}{e^{\frac{1}{n} x}}=0,
$$

and that $F_{n}(0)=0$. Then

$$
\left\langle e_{n} F_{n}, e_{n}\right\rangle=\frac{n}{2} \int_{0}^{\infty} e^{-\frac{2}{n} x} F_{n}^{\prime}(x) d x=\frac{n}{2} \int_{0}^{\infty} e^{-\frac{2}{n} x} \operatorname{sinc}(x) e^{\frac{1}{n} x} e^{-i x} d x
$$




$$
=\frac{n}{2} \int_{0}^{\infty} e^{-\frac{1}{n} x} \operatorname{sinc}(x) \cos (x) d x-i \frac{n}{2} \int_{0}^{\infty} e^{-\frac{1}{n} x} \operatorname{sinc}(x) \sin (x) d x,
$$

which, by computations similar as above involving the Laplace transform, equals

$$
\frac{n}{2}\left\{\frac{\pi}{2}-\arctan \left(\frac{1}{n}\right)-i \frac{1}{4} \ln \left(1+4 n^{2}\right)\right\}=\frac{n}{2} \bar{\lambda}_{n} .
$$

Then

$$
\begin{gathered}
\left\langle P_{I} Q_{J} P_{I} e_{n}, e_{n}\right\rangle=\frac{1}{\pi}\left\langle e_{n}\left(F_{n}+\lambda_{n}\right), e_{n}\right\rangle=\frac{1}{\pi}\left\{\lambda_{n}\left\|e_{n}\right\|_{2}^{2}+\left\langle F_{n} e_{n}, e_{n}\right\rangle\right\} \\
=\frac{1}{\pi}\left\{\frac{n}{2} \lambda_{n}+\frac{n}{2} \bar{\lambda}_{n}\right\}=\frac{n}{\pi} \operatorname{Re}\left(\lambda_{n}\right)=\frac{n}{\pi}\left\{\frac{\pi}{2}-\arctan \left(\frac{1}{n}\right)\right\} .
\end{gathered}
$$

Then

$$
\begin{gathered}
\left\|P_{I} Q_{J} P_{I} e_{n}-e_{n}\right\|_{2}^{2}=\left\|P_{I} Q_{J} P_{I} e_{n}\right\|_{2}^{2}+\left\|e_{n}\right\|_{2}^{2}-2 \operatorname{Re}\left\langle P_{I} Q_{J} P_{I} e_{n}, e_{n}\right\rangle \\
\leq 2\left\|e_{n}\right\|_{2}^{2}-2 \frac{n}{\pi}\left\{\frac{\pi}{2}-\arctan \left(\frac{1}{n}\right)\right\}=2 \frac{n}{\pi} \arctan \left(\frac{1}{n}\right) .
\end{gathered}
$$

Therefore

$$
\frac{1}{\left\|e_{n}\right\|_{2}^{2}}\left\|P_{I} Q_{J} P_{I} e_{n}-e_{n}\right\|_{2}^{2} \leq \frac{4}{\pi} \arctan \left(\frac{1}{n}\right) \rightarrow 0 .
$$

Proposition 3.3. If $I=[0,+\infty)$ and $J=[-1,1]$, then $P_{I} Q_{J} P_{I}$ is non compact, with

$$
\left\|P_{I} Q_{J} P_{I}\right\|=\left\|P_{I} Q_{J}\right\|=1 \text { and }\left\|P_{I}-Q_{J}\right\|=1 .
$$

Proof. If $P_{I} Q_{J} P_{I}$ were compact, there would exist a subsequence $f_{k}=\frac{1}{\left\|e_{n_{k}}\right\|_{2}} e_{n_{k}}$ such that $P_{I} Q_{J} P_{I} f_{k}$ is convergent. By the above lemma, this would imply that the sequence $f_{k}$ is convergent. This is clearly not the case. For instance,

$$
\left\langle f_{k}, f_{k+1}\right\rangle=\frac{1}{\left\|e_{n_{k}}\right\|\left\|e_{n_{k+1}}\right\|}\left\langle e_{n_{k}}, e_{n_{k+1}}\right\rangle=\frac{2}{n_{k}^{1 / 2} n_{k+1}^{1 / 2}} \int_{0}^{\infty} e^{-\left(\frac{1}{n_{k}}+\frac{1}{n_{k+1}}\right) x} d x=\frac{n_{k}^{1 / 2} n_{k+1}^{1 / 2}}{n_{k}+n_{k+1}}
$$

which is less than $\frac{1}{2}$ by the geometric-arithmetic inequality. This clearly implies that the sequence of the unit vectors $f_{k}$ cannot be convergent.

The last assertions follow from the above lemma.

Remark 3.4. Note that Example 3.1 above shows, in particular, that the Volterra-like integral operator

$$
B f(x)=\int_{0}^{x} \operatorname{sinc}(x-t) f(t) d t
$$

is unbounded in $L^{2}(0,+\infty)$ (though it is a Volterra operator on any finite interval $[0, r]$, thus compact with trivial spectrum in $L^{2}(0, r)$, for $\left.r<\infty\right)$. Indeed, if it were bounded, then $T=$ $P_{I} Q_{J} P_{I}-B$

$$
T f(x)=\int_{x}^{\infty} \operatorname{sinc}(x-t) f(t) d t
$$

would be bounded. But the computations above show that the functions $e_{n}(x)=e^{\left(-\frac{1}{n}+i\right) x}$ are eigenfunctions for $T$, with unbounded eigenvalues $\lambda_{n}$. 
Example 3.5. Let $\mathcal{H}=L^{2}(\mathbb{T}, d t)$ where $\mathbb{T}$ is the 1-torus, and consider the decomposition

$$
\mathcal{H}=\mathcal{H}_{-} \oplus \mathcal{H}_{+},
$$

where $\mathcal{H}_{+}$is the Hardy space. Let $\varphi, \psi$ be continuous functions in $\mathbb{T}$ with $\left|\varphi\left(e^{i t}\right)\right|=\left|\psi\left(e^{i t}\right)\right|=1$ for all $t$, and

$$
P=P_{\varphi \mathcal{H}_{+}}^{\perp}=1-P_{\varphi \mathcal{H}_{+}}, \quad Q=P_{\psi \mathcal{H}_{+}} .
$$

Since $\varphi$ and $\psi$ are unimodular, the multiplication operators $M_{\varphi}, M_{\psi}$ are unitary in $\mathcal{H}$ and thus

$$
P Q=M_{\varphi} P_{-} M_{\bar{\varphi} \psi} P_{+} M_{\bar{\psi}}
$$

Note that $\left.P_{-} M_{\bar{\varphi} \psi}\right|_{\mathcal{H}_{+}}=H(\bar{\varphi} \psi)$ is the Hankel operator with symbol $\bar{\varphi} \psi$, which is compact by Hartman's theorem [16] (see also Theorem 5.5 in [20]). Thus $T=P Q$ is compact, and therefore S-decomposable.

Again using Proposition 2.7, one obtains non compact S-decomposable examples. For instance, put now

$$
P=P_{\varphi \mathcal{H}_{+}}, \quad Q=P_{\psi \mathcal{H}_{+}}
$$

In this case

$$
P Q=M_{\varphi} P_{+} M_{\bar{\varphi} \psi} P_{+} M_{\bar{\psi}}
$$

is decomposable Thus the operator $P_{+} M_{\bar{\varphi} \psi} P_{+}$is non-compact and S-decomposable in $L^{2}(\mathbb{T})$. Since it acts non trivially in $\mathcal{H}_{+}$, it follows that the Toeplitz operator $T_{\bar{\varphi} \psi}$ is S-decomposable in $\mathcal{H}_{+}$.

On the other hand, using Theorem 2.2, it follows that

$$
A=P_{\varphi \mathcal{H}_{+}}-P_{\psi \mathcal{H}_{+}}
$$

diagonalizable. In [5] it was shown that \pm 1 are eigenvalues of $A$ only if the winding numbers of $\varphi$ and $\psi$ do not coincide. The other eigenvalues of $A$ are $\pm\left(1-s_{n}^{2}\right)^{1 / 2}$, where $s_{n}$ are the singular values of $T_{\bar{\varphi} \psi}$, and 0 . Since this operator has closed range (being a Fredholm operator), the eigenvalues do not accumulate at \pm 1 . The nullspace of $A$ is infinite dimensional, it contains the subspace $\varphi \psi \mathcal{H}_{+}$.

Again, using Theorem 2.1, one obtains that, with the above hypothesis on $\varphi$ and $\psi$, there exist orthonormal bases $\left\{f_{n}\right\}$ and $\left\{g_{n}\right\}$ of $\mathcal{H}_{+}$such that $\left\langle\varphi f_{n}, \psi g_{m}\right\rangle=0$ if $n \neq m$.

In [18] (Theorem 5.2) J.S. Howland proved that if the function $f$ on $\mathbb{T}$ is $C^{2}$ on the complement of a finite set $\left\{z_{1}, \ldots, z_{n}\right\}$ at which the lateral limits $f\left(z_{i}^{ \pm}\right)$and $f^{\prime}\left(z_{i}^{ \pm}\right)$exist, and one defines the jump of $f$ at $z$ to be

$$
j(z)=f\left(z^{+}\right)-f\left(z^{-}\right),
$$

then the absolutely continuous part of the Hankel operator $H(f)$ is unitarily equivalent to

$$
\oplus_{i=1}^{n} M_{i, z}
$$

where $M_{i, z}$ denotes the operator of multiplication by the variable $z$ in $L^{2}\left(-\frac{1}{2} j\left(z_{i}\right), \frac{1}{2} j\left(z_{i}\right)\right)$. In particular, this implies that if $\bar{\varphi} \psi$ is piecewise $C^{2}$ with jumps as $f$ above, then $P Q$ can be decomposed as a finite direct sum of operators, some of which are multiplication by the variable in $L^{2}$ of an interval. Clearly these operators are not S-decomposable. Then $P Q$ is not Sdecomposable. 
Example 3.6. Let $\mathcal{H}=\mathcal{L} \times \mathcal{S}, B: \mathcal{S} \rightarrow \mathcal{L}$ a bounded operator, and $E=E_{B}$ the idempotent operator given by the matrix

$$
E=\left(\begin{array}{cc}
1_{\mathcal{L}} & B \\
0 & 0
\end{array}\right)
$$

Any idempotent in $\mathcal{B}(\mathcal{H})$ can be expanded in this form. In [1] the reader can find a study of the properties of $E$ in terms of those of $B$. Consider $P=P_{R(E)}=P_{\mathcal{L}}$ and $Q=P_{N(E)}$ and $T=P Q$. Straightforward computations show that $R(E)=\mathcal{L}$ and that

$$
P_{N(E)}=(1-E)\left(1-E-E^{*}\right)^{-1}=\left(\begin{array}{cc}
B B^{*}\left(1+B B^{*}\right)^{-1} & -B\left(1+B^{*} B\right)^{-1} \\
-B^{*}\left(1+B B^{*}\right)^{-1} & \left(1+B^{*} B\right)^{-1}
\end{array}\right) .
$$

Then

$$
T T^{*}=P Q P=\left(\begin{array}{cc}
B B^{*}\left(1+B B^{*}\right)^{-1} & 0 \\
0 & 0
\end{array}\right)
$$

Apparently, $T$ is S-decomposable if and only if $B B^{*}\left(1+B B^{*}\right)^{-1}$ is diagonalizable, which is equivalent to $B B^{*}$ being diagonalizable, or $B$ S-decomposable. Note also that $T$ is compact if and only if $B$ is compact.

If one applies Theorem 2.1 to this example, one obtains that $B$ is S-decomposable if and only if there exist orthonormal bases $\left\{\left(0, v_{n}\right)\right\}$ of $\{0\} \times \mathcal{L}$ and $\left\{\left(w_{n}, B w_{n}\right)\right\}$ of the graph of $B$, such that $\left\langle\left(0, v_{n}\right),\left(w_{m}, B w_{m}\right)\right\rangle=\left\langle v_{n}, B w_{m}\right\rangle=0$ if $n \neq m$. This fact can be proved straightforwardly.

\section{Moore-Penrose pseudoinverses}

Penrose [21] and Greville [13] proved that, for $n \times n$ square matrices, the Moore-Penrose inverse of an idempotent matrix $E$ is a product of orthogonal projections. More precisely, it holds that

$$
E^{\dagger}=P_{N(E) \perp} P_{R(E)} .
$$

Since for matrices $\left(A^{\dagger}\right)^{\dagger}=A$, Penrose-Greville theorem can be stated as follows: an $n \times n$ matrix $E$ is idempotent if and only if $E^{\dagger}$ is a product of two orthogonal projections. This result was extended to infinite dimensional Hilbert space operators in [7], provided that $P Q$ is supposed to have closed range. In the case that $R(P Q)$ is not closed, there is still a similar characterization, but one needs to define the Moore-Penrose inverse for certain unbounded operators. The reader is referred to [8]. As in example 3.6, if $E$ is an idempotent operator, in terms of the decomposition $\mathcal{H}=R(E) \oplus R(E)^{\perp}$, one has

$$
E=\left(\begin{array}{cc}
1 & B \\
0 & 0
\end{array}\right)
$$

where $B: R(E)^{\perp} \rightarrow R(E)$.

Combining the above facts and previous results we obtain the following:

Corollary 4.1. Let $E \in \mathcal{B}(\mathcal{H})$ be an idempotent operator. Then the following are equivalent:

1. $E$ is S-decomposable

2. B is S-decomposable

3. $P_{N(E) \perp} P_{R(E)}$ is $S$-decomposable. 
4. $P_{N(E)} P_{R(E)}$ is S-decomposable.

5. $P_{R(E)}-P_{N(E)}$ is diagonalizable.

6. $P_{R(E)}+P_{N(E)}$ is diagonalizable.

7. There exist orthonormal bases $\left\{\eta_{n}\right\}$ of $R(E)$ and $\left\{\nu_{n}\right\}$ of $N(E)$ such that $\left\langle\eta_{n}, \nu_{m}\right\rangle=0$ if $n \neq m$.

Some of these conditions were proven in [1].

Remark 4.2. By a theorem by Buckholtz ([6], Theorem 1), since $\mathcal{H}$ is the direct sum of $R(E)$ and $N(E)$, it follows that $P_{R(E)}-P_{N(E)}$ is invertible for every idempotent $E$, which in turn implies that $P_{R(E)}+P_{N(E)}$ is invertible. In fact, for any $P, Q \in \mathcal{P}(\mathcal{H}), P-Q$ is invertible if and only if $\|P Q\|<1$ and $\|(1-P)(1-Q)\|<1$, while $P+Q$ is invertible if and only if $\|(1-P)(1-Q)\|<1$. In geometric terms, $\|P Q\|$ is the cosine of the (Dixmier) angle between $R(P)$ and $(Q)$, and $\|(1-P)(1-Q)\|$ is the cosine of the angle between $N(P)$ and $N(Q)$. If $\mathcal{H}$ is the direct sum of $R(P)$ and $R Q$ ), these angles coincide and are not zero.

Finally, note that if $T$ is S-decomposable with expansion $T=\sum_{n \geq 1} \mathcal{S}_{n}\left\langle\quad, \xi_{n}\right\rangle \psi_{n}$, then

$$
T^{\dagger}=\sum_{n \geq 1} \frac{1}{s_{n}}\left\langle\quad, \psi_{n}\right\rangle \xi_{n} .
$$

\section{Isometries}

Given a subspace $\mathcal{S} \subset \mathcal{H}$ with a given orthonormal basis $\mathbf{B}_{\mathcal{S}}=\left\{\xi_{n}: b \geq 1\right\}$, an isometry is defined,

$$
X_{\mathbf{B}_{\mathcal{S}}}: \ell^{2} \rightarrow \mathcal{H}, \quad X_{\mathbf{B}_{\mathcal{S}}}\left(\left\{x_{n}\right\}\right)=\sum_{n \geq 1} x_{n} \xi_{n},
$$

whose range is $\mathcal{S}$. Observe that, by definition, the set of all S-decomposable operators in $\mathcal{H}$ can be described as

$$
\left\{X D Y^{*}: X, Y \text { isometries } \ell^{2} \rightarrow \mathcal{H}, D \in \mathcal{B}\left(\ell^{2}\right) \text { diagonal with positive entries }\right\} .
$$

The condition of bi-orthogonality of Theorem 2.1 can be written in terms of the corresponding isometries.

Proposition 5.1. Let $\mathcal{S}, \mathcal{T}$ be closed subspaces of $\mathcal{H}$. Then $T=P_{\mathcal{S}} P_{\mathcal{T}}$ is $S$-decomposable if and only if there exist isometries $X, Y: \ell^{2} \rightarrow \mathcal{H}$, with range $\mathcal{S}$ and $\mathcal{T}$, respectively, such that

$$
X^{*} Y \in \mathcal{B}\left(\ell^{2}\right)
$$

is a diagonal matrix.

Proof. Suppose that $T$ is decomposable, then by Theorem (2.1), there exist orthonormal bases $\mathbf{B}_{\mathcal{S}}=\left\{\xi_{k}: k \geq 1\right\}$ and $\mathbf{B}_{\mathcal{T}}=\left\{\psi_{n}: n \geq 1\right\}$ of $\mathcal{S}$ and $\mathcal{T}$ such that $\left\langle\xi_{n}, \psi_{k}\right\rangle=0$ if $n \neq k$. Consider the isometries

$$
X=X_{\mathbf{B}_{\mathcal{S}}} \text { and } Y=X_{\mathbf{B}_{\mathcal{T}}} .
$$


Then

$$
X^{*} Y\left(\left\{x_{n}\right\}\right)=\left\{\left\langle\psi_{n}, \xi_{n}\right\rangle x_{n}\right\},
$$

i.e. $X^{*} Y$ is a diagonal matrix whose entries are $\left\langle\psi_{n}, \xi_{n}\right\rangle$.

Conversely, suppose that $X, Y: \ell^{2} \rightarrow \mathcal{H}$ are isometries with $R(X)=\mathcal{S}$ and $R(Y)=\mathcal{T}$, such that $X^{*} Y$ is a diagonal matrix. Denote by $\left\{e_{n}: n \geq 1\right\}$ the canonical basis of $\ell^{2}$. Then $\xi_{n}=X\left(e_{n}\right)$ and $\psi_{k}=Y\left(e_{k}\right)$ form orthonormal bases of $\mathcal{S}$ and $\mathcal{T}$. Moreover

$$
\left\langle\xi_{n}, \psi_{k}\right\rangle=\left\langle X\left(e_{n}\right), Y\left(e_{k}\right)\right\rangle=\left\langle e_{n}, X^{*} Y\left(e_{k}\right)\right\rangle=0 \text { if } n \neq k .
$$

\section{Davis' symmetry}

Let $P, Q$ be projections, and consider

$$
\mathcal{H}^{\prime}=\{R(P) \cap N(Q) \oplus N(P) \cap R(Q)\}^{\perp} .
$$

This subspace reduces $P$ and $Q$, denote by $P^{\prime}=\left.P\right|_{\mathcal{H}^{\prime}}$ and $Q^{\prime}=\left.Q\right|_{\mathcal{H}^{\prime}}$, as operators acting in $\mathcal{H}^{\prime}$. Note that

$$
N(P+Q-1)=N(P-(1-Q))=R(P) \cap N(Q) \oplus N(P) \cap R(Q),
$$

and thus $S^{\prime}=P^{\prime}+Q^{\prime}-1$ is a selfadjoint operator with trivial kernel (and thus dense range) in $\mathcal{H}^{\prime}$. Let

$$
S^{\prime}=V\left|S^{\prime}\right|
$$

be the polar decomposition. It follows that $V$ is a selfadjoint unitary operator, i.e., a symmetry. The fact that

$$
S^{\prime} P^{\prime}=Q^{\prime} P^{\prime}=Q^{\prime} S^{\prime} \quad\left(\text { also } S^{\prime} Q^{\prime}=P^{\prime} Q^{\prime}=P^{\prime} S^{\prime}\right)
$$

implies that the symmetry $V$ intertwines $P^{\prime}$ and $Q^{\prime}$ :

$$
V P^{\prime} V=Q^{\prime}, V Q^{\prime} V=P^{\prime}
$$

Also one recovers $P^{\prime}$ and $Q^{\prime}$ in terms of $V$ and the difference $A=P^{\prime}-Q^{\prime}$, by means of the formulas of the previous section:

$$
P^{\prime}=P_{V}, \quad Q^{\prime}=Q_{V}
$$

These facts were proved by Chandler Davis in [10]. Then $T=P Q$, in the decomposition $\mathcal{H}=\mathcal{H}^{\prime \perp} \oplus \mathcal{H}^{\prime}$ is given by

$$
T=0 \oplus V Q^{\prime} V Q^{\prime}=0 \oplus P^{\prime} V P^{\prime} V .
$$

The following result is a straightforward consequence of the results in the previous section:

Proposition 6.1. $T=P Q$ is $S$-decomposable if and only $Q^{\prime} V Q^{\prime}$ is diagonalizable (equivalently: $P^{\prime} V P^{\prime}$ is diagonalizable). If $\left\{\xi_{n}\right\}$ is an orthonormal system of eigenvectors for $Q^{\prime} V Q^{\prime}$, then $\left\langle V \xi_{n}, \xi_{k}\right\rangle=0$ if $n \neq k$. 
Proof. If $Q^{\prime} V Q^{\prime}=\sum_{n \geq 1} \lambda_{n}\left\langle, \xi_{n}\right\rangle \xi_{n}$, then

$$
P^{\prime} Q^{\prime}=V Q^{\prime} V Q^{\prime}=\sum_{n \geq 1} \lambda_{n}\left\langle\quad, \xi_{n}\right\rangle V \xi_{n}
$$

and thus the orthonormal systems $\left\{\xi_{n}\right\}$ and $\left\{V \xi_{n}\right\}$ are bi-orthogonal.

Remark 6.2. Suppose that

$$
P^{\prime} Q^{\prime}=V Q^{\prime} V Q^{\prime}=\sum_{n \geq 1} s_{n}\left\langle\quad, \xi_{n}\right\rangle \psi_{n}
$$

Then

$$
Q^{\prime} V Q^{\prime}=\sum_{n \geq 1} s_{n}\left\langle\quad, \xi_{n}\right\rangle V \psi_{n}=\sum_{n \geq 1} s_{n}\left\langle\quad, V \psi_{n}\right\rangle \xi_{n} .
$$

In particular, if all the singular values have multiplicity 1, then $V \psi_{n}= \pm \xi_{n}$.

Davis' symmetry is related to the metric geometry of the set $\mathcal{P}(\mathcal{H})$ of projections in $\mathcal{H}$ (also called Grassmannian manifold of $\mathcal{H})$. If one measures the length of a continuous piecewise smooth curve $p(t) \in \mathcal{P}(\mathcal{H}), t \in I$, by means of

$$
\ell(p)=\int_{I}\left\|\frac{d}{d t} p(t)\right\| d t
$$

it was shown $([22],[9])$ that curves in $\mathcal{P}(\mathcal{H})$ of the form

$$
P(t)=e^{i t X} P e^{-i t X}
$$

for $X^{*}=X$ with $\|X\| \leq \pi / 2$, such that $X$ is $P$-codiagonal (i.e $P X P=P^{\perp} X P^{\perp}=0$ ) have minimal length along their paths for $|t| \leq 1$. That is, any curve joining a pair of projections in this path cannot be shorter that the part of $P(t)$ which joins these projections. Given two projections $P, Q$, in [2] it was shown that there exists a unique $X\left(X^{*}=X,\|X\| \leq \pi / 2, X\right.$ is $P$-codiagonal) such that $e^{i X} P e^{-i X}=Q$ if and only if

$$
N(P+Q-1)=\{0\} .
$$

Let us denote $X=X_{P, Q}$ if such is the case. Also in [2] it was shown that $V$ and $X_{P, Q}$ are related by

$$
V=e^{i X_{P, Q}}(2 P-1) .
$$

Note that since (always in the case $N(P+Q-1)=\{0\})\left\|X_{P, Q}\right\| \leq \pi / 2, X_{P, Q}$ is obtained from $V$ by means of the usual $\log$ function:

$$
X_{P, Q}=-i \log (V(2 P-1)) .
$$

Define the geodesic distance $d(P, Q)$ in $\mathcal{P}(\mathcal{H})$ as

$$
d(P, Q)=\inf \{\ell(p): p \text { joins } P \text { and } Q \text { in } \mathcal{P}(\mathcal{H})\},
$$

Porta and Recht proved in [22] that

$$
d(P, Q)=\left\|X_{P, Q}\right\|
$$


Remark 6.3. Formula (2) has a geometric interpretation. The fact that $X_{P, Q}$ is $P$-codiagonal, is equivalent to saying that $X_{P, Q}$ and $2 P-1$ anti-commute, it follows that $e^{i t X_{P, Q}}(2 P-1)=$ $(2 P-1) e^{-i t X_{P, Q}}$. Then, in particular $V=e^{\frac{i}{2} X_{P, Q}}(2 P-1) e^{-\frac{i}{2} X_{P, Q}}$, or equivalently

$$
\frac{1}{2}(1+V)=e^{\frac{i}{2} X_{P, Q}} P e^{-\frac{i}{2} X_{P, Q}}
$$

In other words, the projection $\frac{1}{2}(1+V)$ (onto the eigenspace where the symmetry $V$ acts as the identity) is the midpoint of the geodesic $P(t)$ joining $P$ and $Q$.

From the above facts, the following is apparent:

Corollary 6.4. Let $P, Q$ be projections and, as above, $P^{\prime}, Q^{\prime}$ the respective reductions to $N(P+$ $Q-1)^{\perp}$, and let $V$ be Davis' symmetry induced by these. Then

$$
P^{\prime} V P^{\prime}=P^{\prime} e^{X_{P^{\prime}, Q^{\prime}} P^{\prime}} \quad \text { and } \quad Q^{\prime} V Q^{\prime}=Q^{\prime} e^{-X_{P^{\prime}, Q^{\prime}} Q^{\prime}} .
$$

Thus $P Q$ is S-decomposable if and only if $P^{\prime} e^{X_{P^{\prime}, Q^{\prime}} P^{\prime}}$ is diagonalizable.

Proof. Since $V=e^{i X_{P^{\prime}, Q^{\prime}}\left(2 P^{\prime}-1\right) \text {, then }}$

$$
P^{\prime} V P^{\prime}=P^{\prime} e^{i X_{P^{\prime}, Q^{\prime}}\left(2 P^{\prime}-1\right) P^{\prime}}=P^{\prime} e^{i X_{P^{\prime}, Q^{\prime}} P^{\prime}} .
$$

Similarly, $V=P^{\prime} e^{i X_{P^{\prime}, Q^{\prime}}}=e^{-i X_{P^{\prime}, Q^{\prime}} Q^{\prime}}$, and so forth.

Remark 6.5. Since $Q^{\prime}=e^{i X_{P^{\prime}, Q^{\prime}} P^{\prime}} e^{-i X_{P^{\prime}, Q^{\prime}}}\left(2 P^{\prime}-1\right) P^{\prime}$, it also follows that

$$
P^{\prime} e^{i X_{P^{\prime}, Q^{\prime}} P^{\prime}}=P^{\prime} Q^{\prime} e^{-i X_{P^{\prime}, Q^{\prime}}}=e^{-i X_{P^{\prime}, Q^{\prime}} Q^{\prime} P^{\prime}}
$$

and

$$
Q^{\prime} e^{-i X_{P^{\prime}, Q^{\prime}} Q^{\prime}}=Q^{\prime} P^{\prime} e^{i X_{P^{\prime}, Q^{\prime}}}=e^{i X_{P^{\prime}, Q^{\prime}} P^{\prime} Q^{\prime}} .
$$

Remark 6.6. If the matrix of $X_{P^{\prime}, Q^{\prime}}$ in terms of $P^{\prime}$ is given by

$$
X_{P^{\prime}, Q^{\prime}}=\left(\begin{array}{cc}
0 & Z \\
Z^{*} & 0
\end{array}\right)
$$

then

$$
P^{\prime} V P^{\prime}=P^{\prime} e^{i X_{P^{\prime}, Q^{\prime}} P^{\prime}}=\left(\begin{array}{cc}
\cos \left(\left|Z^{*}\right|\right) & 0 \\
0 & \cos (|Z|)
\end{array}\right)
$$

From this last remark, it follows that

Theorem 6.7. $P Q$ is $S$-decomposable if and only if $Z$ is $S$-decomposable, if and only if $X_{P^{\prime}, Q^{\prime}}$ is diagonalizable.

Proof.

$$
X_{P^{\prime}, Q^{\prime}}^{2}=\left(\begin{array}{cc}
Z Z^{*} & 0 \\
0 & Z^{*} Z
\end{array}\right)
$$

Thus $X_{P^{\prime}, Q^{\prime}}$ is diagonalizable if and only if $Z$ is $\mathrm{S}$-decomposable. Indeed, if $Z$ is S-decomposable,

$$
Z=\sum_{n \geq 1} s_{n}\left\langle, v_{n}\right\rangle w_{n}, Z^{*}=\sum_{n \geq 1} s_{n}\left\langle, w_{n}\right\rangle v_{n}
$$


Note that $\left\{v_{n}\right\}$ span $R\left(P^{\prime}\right)$ and $\left\{w_{n}\right\}$ span $R\left(P^{\prime}\right)^{\perp}$, therefore, they are pairwise orthogonal systems of vectors. Then

$$
X_{P^{\prime}, Q^{\prime}} v_{n}=s_{n} w_{n} \text { and } \quad X_{P^{\prime}, Q^{\prime}} w_{n}=s_{n} v_{n}
$$

For each fixed $n$, the two dimensional space generated by $v_{n}$ and $w_{n}$ reduces $X_{P^{\prime}, Q^{\prime}}$. As in a previous argument, $X_{P^{\prime}, Q^{\prime}}$ can be diagonalized in each of these spaces, providing a diagonalization of the whole operator $X_{P^{\prime}, Q^{\prime}}$. The converse statement is apparent.

Finally, let us further exploit formula (2).

Corollary 6.8. If $A^{\prime}=P^{\prime}-Q^{\prime}$, then

$$
e^{i X_{P^{\prime}, Q^{\prime}}}=V A^{\prime}+\left(1-A^{\prime 2}\right)^{1 / 2} .
$$

Proof. In $N(P+Q-1)^{\perp}, P^{\prime}=P_{V}=\frac{1}{2}\left\{1+A^{\prime}+V\left(1-A^{\prime 2}\right)^{1 / 2}\right\}$, thus

$$
e^{i X_{P^{\prime}, Q^{\prime}}}=V\left(2 P^{\prime}-1\right)=V\left\{A^{\prime}+V\left(1-A^{\prime 2}\right)^{1 / 2}\right\}=V A^{\prime}+\left(1-A^{\prime 2}\right)^{1 / 2} .
$$

In particular, if $P Q$ is $\mathrm{S}$-decomposable, with singular values of simple multiplicity, one has the following

Theorem 6.9. Let $P Q$ be S-decomposable, $P^{\prime} Q^{\prime}=\sum_{n \geq 1} s_{n}\left\langle\quad, \xi_{n}\right\rangle \psi_{n}$, with $s_{n}$ of multiplicity 1. Then $X_{P^{\prime}, Q^{\prime}}$ is diagonalized as follows

$$
\left.X_{P^{\prime} Q^{\prime}}=\sum_{n \geq 1} i \log \left(s_{n}+i\left(1-s_{n}^{2}\right)^{1 / 2}\right) \eta_{n} \otimes \eta_{n}+i \log \left(s_{n}-i\left(1-s_{n}^{2}\right)^{1 / 2}\right)\right) \zeta_{n} \otimes \zeta_{n},
$$

where

$$
\eta_{n}=\frac{1}{\sqrt{2}} \nu_{n}-\frac{i}{\sqrt{2}} \omega_{n} \quad \text { and } \quad \zeta_{n}=\frac{1}{\sqrt{2}} \nu_{n}+\frac{i}{\sqrt{2}} \omega_{n}
$$

and (as in the proof of Theorem 2.2)

$$
\nu_{n}=\left(\left(1-s_{n}^{2}\right)^{1 / 2}-1\right) \xi_{n}+s_{n} \psi_{n} \quad \text { and } \quad \omega_{n}=\left(-\left(1-s_{n}^{2}\right)^{1 / 2}-1\right) \xi_{n}+s_{n} \psi_{n}
$$

Proof. If $P Q$ is S-decomposable, considering the decomposition of $\left.P Q\right|_{N(P+Q-1)^{\perp}}=P^{\prime} Q^{\prime}$, in the proof of Theorem 2.2,

$$
A^{\prime}=\sum_{n \geq 1}\left(1-s_{n}^{2}\right) \nu_{n} \otimes \nu_{n}-\left(1-s_{n}^{2}\right)^{1 / 2} \omega_{n} \otimes \omega_{n}
$$

for $\nu_{n}, \omega_{n}$ described above. Then

$$
\left(1-A^{\prime 2}\right)^{1 / 2}=\sum_{n \geq 1} s_{n} \nu_{n} \otimes \nu_{n}+s_{n} \omega_{n} \otimes \omega_{n} .
$$

Recall that $V A=-V A$, or equivalently, $V A V=-A$ (see remarks before Theorem 2.4). Note that in $N(P+Q-1)^{\perp}$ we have erased the eigenvalues \pm 1 from $A$. Then, using Theorem 2.2, 
the fact that the singular values of $P^{\prime} Q^{\prime}$ have simple multiplicity implies that the (non nil) eigenvalues of $A^{\prime}$ have single multiplicity. These two assertions imply that

$$
V \nu_{n} \otimes V \nu_{n}=V\left(\nu_{n} \otimes \nu_{n}\right) V=\omega_{n} \otimes \omega_{n} .
$$

Thus, in the diagonalization of $A^{\prime}$, we may replace $\xi_{n}, \psi_{n}$ by scalar multiples (of modulus one) in order that

$$
V \nu_{n}=\omega_{n} \text { and } V \omega_{n}=\nu_{n}
$$

Then

$$
V A^{\prime}=\sum_{n \geq 1}\left(1-s_{n}^{2}\right)^{1 / 2} \omega_{n} \otimes \nu_{n}-\left(1-s_{n}^{2}\right)^{1 / 2} \nu_{n} \otimes \omega_{n}
$$

Thus, by the formula in the above Corollary,

$e^{i X_{P^{\prime}, Q^{\prime}}}=V A^{\prime}+\left(1-A^{\prime 2}\right)^{1 / 2}=\sum_{n \geq 1}\left(1-s_{n}^{2}\right)^{1 / 2} \omega_{n} \otimes \nu_{n}-\left(1-s_{n}^{2}\right)^{1 / 2} \nu_{n} \otimes \omega_{n}+s_{n} \nu_{n} \otimes \nu_{n}+s_{n} \omega_{n} \otimes \omega_{n}$.

Note that this is a block diagonal operator, with $2 \times 2$ blocks, given by the subspaces generated by the (orthonormal) vectors $\nu_{n}$ and $\omega_{n}$ for each $n$. Each block, in this basis, is given by

$$
\left(\begin{array}{cc}
s_{n} & -\left(1-s_{n}^{2}\right)^{1 / 2} \\
\left(1-s_{n}^{2}\right)^{1 / 2} & s_{n}
\end{array}\right),
$$

whose eigenvalues are $s_{n}+i\left(1-s_{n}^{2}\right)^{1 / 2}$ and $s_{n}-i\left(1-s_{n}^{2}\right)^{1 / 2}$, with (orthonormal) eigenvectors

$$
\eta_{n}=\frac{1}{\sqrt{2}} \nu_{n}-\frac{i}{\sqrt{2}} \omega_{n} \text { and } \zeta_{n}=\frac{1}{\sqrt{2}} \nu_{n}+\frac{i}{\sqrt{2}} \omega_{n}
$$

respectively, and the proof follows.

Note that since $0<s_{n}$, the logarithms of these eigenvalues have modulus smaller than $\pi / 2$, a fact predicted by the condition $\left\|X_{P^{\prime}, Q^{\prime}}\right\| \leq \pi / 2$.

Examples 6.10. Let us review the examples in Section 3:

1. For $I, J \subset \mathbb{R}^{n}$ of finite Lebesgue measure, it is known (see [19], [12]) that

$$
N\left(P_{I}+Q_{J}-1\right)=\{0\} .
$$

Thus $P_{I}^{\prime}=P_{I}$ and $Q_{J}^{\prime}=Q_{J}$. It is also known (see for instance [17]) that in the particular case when $I$ and $J$ are intervals, the singular values of of $P_{I} Q_{J}$ have multiplicity one. Moreover the functions $\psi_{n}$ and $\xi_{n}$ are known to be the prolate spheroidal functions, for precise $I$ and $J$ (intervals in $\mathbb{R}$ ) [17]. It follows that one can compute explicitely the eigenvectors of $X_{P_{I}, Q_{J}}$ for such intervals $I, J$.

2. As in Example 3.5, consider $\mathcal{H}=L^{2}(\mathbb{T})$ and

$$
P=P_{\varphi \mathcal{H}_{+}}, \quad Q=P_{\psi \mathcal{H}_{+}},
$$

for $\varphi, \psi$ continuous functions in $\mathbb{T}$, of modulus 1 . It was shown in [5] that if $\varphi$ and $\psi$ have the same winding number, then

$$
N(P+Q-1)=\varphi \mathcal{H}_{+} \cap\left(\psi \mathcal{H}_{+}\right)^{\perp} \oplus\left(\varphi \mathcal{H}_{+}\right)^{\perp} \cap \psi \mathcal{H}_{+}=\{0\} .
$$


3. As in example 3.6, let $\mathcal{H}=\mathcal{L} \times \mathcal{S}$ and $B: \mathcal{S} \rightarrow \mathcal{L}$ a bounded operator, $P=P_{R(E)}=P_{\mathcal{L}}$ and $Q=P_{N(E)}$ and $T=P Q$. Elementary computations show that

$$
N(P+Q-1)=R(B)^{\perp} \times\{0\} \oplus\{0\} \times N(B) .
$$

Thus this nullspace is trivial if and only if $B$ has trivial nullspace and dense range. Suppose that this is the case. Also it is straightforward to verify that

$$
P+Q-1=\left(\begin{array}{cc}
B B^{*}\left(1+B B^{*}\right)^{-1} & -B\left(1+B^{*} B\right)^{-1} \\
-B^{*}\left(1+B B^{*}\right)^{-1} & -B^{*} B\left(1+B^{*} B\right)^{-1}
\end{array}\right)
$$

and that

$$
(P+Q-1)^{2}=\left(\begin{array}{cc}
B B^{*}\left(1+B B^{*}\right)^{-1} & 0 \\
0 & B^{*} B\left(1+B^{*} B\right)^{-1}
\end{array}\right)
$$

Then

$$
|P+Q-1|=\left(\begin{array}{cc}
\left(B B^{*}\right)^{1 / 2}\left(1+B B^{*}\right)^{-1 / 2} & 0 \\
0 & \left(B^{*} B\right)^{1 / 2}\left(1+B^{*} B\right)^{-1 / 2}
\end{array}\right) .
$$

Thus

$V=(P+Q-1)|P+Q-1|^{-1}=\left(\begin{array}{cc}\left|B^{*}\right|\left(1+\left|B^{*}\right|^{2}\right)^{-1 / 2} & -B|B|^{-1}\left(1+|B|^{2}\right)^{-1 / 2} \\ -B^{*}\left|B^{*}\right|^{-1}\left(1+\left|B^{*}\right|^{2}\right)^{-1 / 2} & -|B|\left(1+|B|^{2}\right)^{-1 / 2}\end{array}\right)$.

This computation is apparent if $B$ (and thus $|P+Q-1|$ ) is invertible, but also makes sense when $B$ has trivial nullspace and dense range. If $B=W|B|=\left|B^{*}\right| W$ are the polar decompositions of $B$, one has

$$
V=\left(\begin{array}{cc}
\left|B^{*}\right|\left(1+\left|B^{*}\right|^{2}\right)^{-1 / 2} & -W\left(1+|B|^{2}\right)^{-1 / 2} \\
-W\left(1+\left|B^{*}\right|^{2}\right)^{-1 / 2} & -|B|\left(1+|B|^{2}\right)^{1 / 2}
\end{array}\right)
$$

where $W\left(1+\left|B^{*}\right|^{2}\right)^{-1 / 2}$ can be replaced by $\left(1+|B|^{2}\right)^{-1 / 2} W^{*}$.

Therefore

$$
e^{i X_{P, Q}}=V(2 P-1)=\left(\begin{array}{cc}
\left|B^{*}\right|\left(1+\left|B^{*}\right|^{2}\right)^{-1 / 2} & W\left(1+|B|^{2}\right)^{-1 / 2} \\
-W\left(1+\left|B^{*}\right|^{2}\right)^{-1 / 2} & |B|\left(1+|B|^{2}\right)^{1 / 2}
\end{array}\right)
$$

Suppose now that $B$ is S-decomposable, $B=\sum_{n \geq 1} s_{n}\left\langle\right.$, $\left.e_{n}\right\rangle f_{n}$, where since $B$ has trivial nullspace and dense range, where $\left\{e_{n}\right\}$ and $\left\{f_{n}\right\}$ are orthonormal bases of $\mathcal{S}$ and $\mathcal{L}$, respectively. Then

$$
|B|=\sum_{n \geq 1} s_{n} e_{n} \otimes e_{n}, \quad\left|B^{*}\right|=\sum_{n \geq 1} s_{n} f_{n} \otimes f_{n},
$$

and $W$ is a unitary operators $(W: \mathcal{S} \rightarrow \mathcal{L})$, with $W e_{n}=f_{n}$. Let $\xi_{n}=\left(e_{n}, 0\right), \psi_{n}=\left(0, f_{n}\right)$. Then $\left\{\xi_{n}, \psi_{n}\right\}$ span a reducing subspace of $T=P Q, P=P_{R(E)}, Q=P_{N(E)}$, and in view of the above formulas, also reducing for $V$ and $X_{P, Q}$. Elementary computations show that the matrix of $e^{i X_{P, Q}}$ in the basis of this reducing subspace is

$$
\frac{1}{\left(1+s_{n}^{2}\right)^{1 / 2}}\left(\begin{array}{cc}
s_{n} & 1 \\
-1 & s_{n}
\end{array}\right)
$$


Let $\theta_{n}$ be defined by $\cos \left(\theta_{n}\right)=\frac{s_{n}}{\left(1+s_{n}^{2}\right)^{1 / 2}}$ and $\sin \left(\theta_{n}\right)=\frac{1}{\left(1+s_{n}^{2}\right)^{1 / 2}}$ (or equivalently, since $\left.s_{n}>0: \tan \left(\theta_{n}\right)=\frac{1}{s_{n}}\right)$, then the matrix of $X_{P, Q}$ in this reducing subspace is

$$
\left(\begin{array}{cc}
0 & -i \theta_{n} \\
i \theta_{n} & 0
\end{array}\right)
$$

Recall [2] that if $P$ and $Q$ are projections such that $N(P+Q-1)=\{0\}$, there exists a unique exponent $X_{P, Q}$ with $d(P, Q)=\left\|X_{P, Q}\right\|$. In particular, one has the following consequence:

Corollary 6.11. Let $B: \mathcal{S} \rightarrow \mathcal{L}$ with trivial nullspace and dense range, and $E$ as in Example 3.6.

(a) If $B$ is invertible, then the geodesic dictance between $P_{R(E)}$ and $P_{N(E)}$ is

$$
d\left(P_{R(E)}, P_{N(E)}\right)=\arctan \left(\left\|B^{-1}\right\|\right)<\pi / 2 .
$$

(b) If $B$ is non invertible (i.e. $B^{-1}$ is unbounded), then

$$
d\left(P_{R(E)}, P_{N(E)}\right)=\pi / 2 .
$$

Proof. Suppose that $B$ is S-decomposable. If $B$ is invertible, $s_{n} \in\left(\left\|B^{-1}\right\|^{-1},\|B\|\right)$, and if $B$ is non invertible there exists a decreasing subsequence $s_{n_{k}}$ of singular values of $B$, such that $s_{n_{k}} \rightarrow 0$. Thus the claims follow from the previous computations.

Suppose now $B$ arbitrary. Clearly $|B|$ can be approximated by positive invertible operators $A_{k}$ with finite spectrum, in particular, diagonalizable. If $B=W|B|$, then $B_{k}=W A_{k}$ approximate $B$ (as in 6.10.3). Since $B$ has trivial nullspace and dense range, $W$ is a unitary operator. Then $B_{k}$ are S-decomposable, with finite singular values (increasingly ordered) $s_{k, i}, 1 \leq i \leq n_{k}$. Note that $P=P_{R(E)}$ and $Q=P_{N(E)}$ are continuous functions of $B$. Denote by $E_{k}, P_{k}=P_{R\left(E_{k}\right)}$ and $Q_{k}=P_{N\left(E_{k}\right)}$ the operators acting in $\mathcal{L} \times \mathcal{S}$ which correspond to $B_{k}$. Then

$$
d\left(P_{k}, Q_{k}\right) \rightarrow d(P, Q) .
$$

From the previous case, $d\left(P_{k}, Q_{k}\right)=\tan ^{-1}\left(\frac{1}{s_{k, 1}}\right)$. If $B$ is invertible, $\frac{1}{s_{k, 1}} \rightarrow\left\|B^{-1}\right\|$. Otherwise, $\frac{1}{s_{k, 1}} \rightarrow \infty$.

Remark 6.12. As mentioned in the beginning of Section 2, if $T=P Q$, there may exist many factorizations, and that there exist a canonical factorization

$$
T=P_{\overline{R(T)}} P_{N(T)^{\perp}}
$$

with the following minimality property: for any $\xi \in \mathcal{H}$, and any other factorization $T=P Q$, one has

$$
\left\|P_{\overline{R(T)}} \xi-P_{N(T) \perp} \xi\right\| \leq\|P \xi-Q \xi\| .
$$

. In [3] it was shown that in example 3.1 the factorization $T=P_{I} Q_{J}$ is canonical. 
In example 3.6 suppose that $B: \mathcal{S} \rightarrow \mathcal{L}$ has trivial nullspace and dense range. Elementary computations show that for $T=P_{R(E)} P_{N(E)}$,

$$
N(T)=R\left(E^{*}\right) \text { and } N\left(T^{*}\right)=N\left(B^{*}\right) \times \mathcal{S}=\{0\} \times \mathcal{S} .
$$

Then $\overline{R(T)}=R(E)$ and $N(T)=N(E)$, and this decomposition is also canonical.

Also in [3], it was shown that $R\left(P_{I}\right)+R\left(Q_{J}\right)$ is a closed proper direct sum, therefore $P_{I} Q_{J}$ is a different example from $P_{R(E)} P_{N(E)}$, for which $R(E)+N(E)$ is the whole space.

\section{Dilations of contractions}

Let $\Gamma$ be a contraction in a Hilbert space $\mathcal{H}_{0}$. P.R. Halmos showed in [14], that $\Gamma$ is the 1,1 corner of a unitary operator $U$ acting in $\mathcal{H}_{0} \times \mathcal{H}_{0}$, namely

$$
U=\left(\begin{array}{cc}
\Gamma & \left(1-\Gamma \Gamma^{*}\right)^{1 / 2} \\
\left(1-\Gamma^{*} \Gamma\right)^{1 / 2} & -\Gamma^{*}
\end{array}\right)
$$

If

$$
P=P_{\Gamma}=\left(\begin{array}{ll}
1 & 0 \\
0 & 0
\end{array}\right) \text { and } Q=Q_{\Gamma}=U^{*} P U=\left(\begin{array}{cc}
\Gamma^{*} \Gamma & \Gamma^{*}\left(1-\Gamma \Gamma^{*}\right)^{1 / 2} \\
\left(1-\Gamma \Gamma^{*}\right)^{1 / 2} \Gamma & 1-\Gamma \Gamma^{*}
\end{array}\right)
$$

then

$$
\left(\begin{array}{ll}
\Gamma & 0 \\
0 & 0
\end{array}\right)=U Q_{\Gamma} P_{\Gamma}
$$

i.e. $\Gamma$ factors as a unitary operator times a product of projections, on a bigger space. Apparently, $\Gamma$ is S-decomposable in $\mathcal{H}$ if and only if $Q P$ is decomposable in $\mathcal{H} \times \mathcal{H}$

Moreover, if

$$
\Gamma=\sum_{n \geq 1} s_{n}\left\langle\quad, \xi_{n}\right\rangle \psi_{n}
$$

then

$$
Q P=\sum_{n \geq 1} s_{n}\left\langle\quad,\left(\begin{array}{c}
\xi_{n} \\
0
\end{array}\right)\right\rangle\left(\begin{array}{c}
s_{n} \xi_{n} \\
\left(1-s_{n}^{2}\right)^{1 / 2} \psi_{n}
\end{array}\right) .
$$

Lemma 7.1. On $\mathcal{H} \times \mathcal{H}$, one has that

$$
R(P) \cap R(Q)=N\left(1-\Gamma^{*} \Gamma\right) \oplus 0, N(P) \cap N(Q)=\{0\} \oplus N\left(1-\Gamma \Gamma^{*}\right)
$$

and

$$
R(P) \cap N(Q)=N(\Gamma) \oplus\{0\}, N(P) \cap R(Q)=\{0\} \oplus N\left(\Gamma^{*}\right) .
$$

Proof. A vector in $R(P)$ is of the form $\xi=\left(\begin{array}{c}\xi_{1} \\ 0\end{array}\right) . Q \xi=\xi$ if and only if

$$
\Gamma^{*} \Gamma \xi_{1}=\xi_{1} \text { and }\left(1-\Gamma \Gamma^{*}\right)^{1 / 2} \Gamma \xi_{1}=0 .
$$

Note that $\left(1-\Gamma \Gamma^{*}\right)^{1 / 2} \Gamma=\Gamma\left(1-\Gamma^{*} \Gamma\right)^{1 / 2}$. Thus $\Gamma^{*} \Gamma \xi_{1}=\xi_{1}$ implies that $\left(1-\Gamma^{*} \Gamma\right)^{1 / 2} \xi_{1}=0$.

A vector $\xi \in R(P)$ belongs to $N(Q)$ if and only if $\Gamma^{*} \Gamma \xi_{1}=0$, i.e., $\xi_{1} \in N(\Gamma)$.

The other two statements are similar. 
Remark 7.2. Straightforward computations show that

$$
(P+Q-1)^{2}=\left(\begin{array}{cc}
\Gamma^{*} \Gamma & 0 \\
0 & \Gamma \Gamma^{*}
\end{array}\right) \text { and thus }|P+Q-1|=\left(\begin{array}{cc}
\left(\Gamma^{*} \Gamma\right)^{1 / 2} & 0 \\
0 & \left(\Gamma \Gamma^{*}\right)^{1 / 2}
\end{array}\right) .
$$

Suppose that $N(\Gamma)=N\left(\Gamma^{*}\right)=\{0\}$ (i.e., $P+Q-1$ has trivial nullspace and dense range). If $\Gamma=W|\Gamma|=\left|\Gamma^{*}\right| W$ are the polar decompositions (with $W$ a unitary operator), then

$V=\left(\begin{array}{cc}|\Gamma| & W^{*}\left(1-\Gamma \Gamma^{*}\right)^{1 / 2} \\ \left(1-\Gamma \Gamma^{*}\right)^{1 / 2} W & -\left|\Gamma^{*}\right|\end{array}\right)$ and $e^{i X_{P, Q}}=\left(\begin{array}{cc}|\Gamma| & -W^{*}\left(1-\Gamma \Gamma^{*}\right)^{1 / 2} \\ \left(1-\Gamma \Gamma^{*}\right)^{1 / 2} W & \left|\Gamma^{*}\right|\end{array}\right)$

With similar computations as above, one sees that if $\Gamma$ is S-decomposable with singular values $0<s_{n} \leq 1$, then the spectrum of $X_{P, Q}$ is $\left\{ \pm \theta_{n}: \cos \left(\theta_{n}\right)=s_{n}\right\}$. With an argument as in Corollary 6.11, one has:

Corollary 7.3. Let $\Gamma$ be a contraction in $\mathcal{H}_{0}$ with trivial nullspace and dense range, and $P_{\Gamma}$, $Q_{\Gamma}$ the above projections in $\mathcal{H}_{0} \times \mathcal{H}_{0}$.

1. If $\Gamma$ is invertible, then

$$
d\left(P_{\Gamma}, Q_{\Gamma}\right)=\cos ^{-1}\left(\left\|\Gamma^{-1}\right\|^{-1}\right) .
$$

2. If $\Gamma$ is non invertible, then

$$
d\left(P_{\Gamma}, Q_{\Gamma}\right)=\pi / 2
$$

\section{References}

[1] Andruchow, E.; Classes of idempotents in Hilbert space. Complex Anal. Oper. Theory 10 (2016), no. 6, 1383-1409.

[2] Andruchow, E.; Operators which are the difference of two projections. J. Math. Anal. Appl. 420 (2014), no. 2, 1634-1653.

[3] Andruchow, E. ; Corach, G. Uncertainty principle and geometry of the infinite Grassmann manifold, arXiv:1701.03733.

[4] Andruchow, E. ; Corach, G. Essentially orthogonal subspaces, J. Operator Theory (to appear), arXiv:1701.03737.

[5] Andruchow, E.; Chiumiento, E.; Larotonda, G. Geometrical significance of Toeplitz kernels, arXiv:1608.05737

[6] Buckholtz, D. Hilbert space idempotents and involutions, Proc. Amer. Math. Soc. 128 (2000), 1415-1418.

[7] Corach, G.; Maestripieri, A. Polar decomposition of oblique projections. Linear Algebra Appl. 433 (2010), 511-519.

[8] Corach, G.; Maestripieri, A. Products of orthogonal projections and polar decompositions. Linear Algebra Appl. 434 (2011), no. 6, 1594-1609. 
[9] Corach, G.; Porta, H.; Recht, L. The geometry of spaces of projections algebras. Adv. Math. 101 (1993), no. 1, 59-77.

[10] Davis, C. Separation of two linear subspaces. Acta Sci. Math. Szeged 19 (1958) 172-187.

[11] Donoho, D. L.; Stark, P. B. Uncertainty principles and signal recovery. SIAM J. Appl. Math. 49 (1989), no. 3, 906-931.

[12] Folland, G. B.; Sitaram, A. The uncertainty principle: a mathematical survey. J. Fourier Anal. Appl. 3 (1997), no. 3, 207-238.

[13] Greville, T. N. E. Solutions of the matrix equation $X A X=X$, and relations between oblique and orthogonal projectors. SIAM J. Appl. Math. 26 (1974), 828-832.

[14] Halmos, P. R. Normal dilations and extensions of operators. Summa Brasil. Math. 2, (1950). 125-134.

[15] Halmos, P. R. Two subspaces. Trans. Amer. Math. Soc. 144 (1969) 381-389.

[16] Hartman, P. On completely continuous Hankel matrices. Proc. Amer. Math. Soc. 91958 $862-866$.

[17] Hogan, J. A.; Lakey, J. D. Time-frequency and time-scale methods. Adaptive decompositions, uncertainty principles, and sampling. Applied and Numerical Harmonic Analysis. Birkhäuser Boston, Inc., Boston, MA, 2005. xxii+388 pp. ISBN: 0-8176-4276-5

[18] Howland, J.S., Spectral theory of selfadjoint Hankel matrices. Michigan Math. J. 33 (1986), no. $2,145-153$.

[19] Lenard, A. The numerical range of a pair of projections. J. Functional Analysis 10 (1972), $410-423$.

[20] Peller, V. V. An excursion into the theory of Hankel operators. Holomorphic spaces (Berkeley, CA, 1995), 65-120, Math. Sci. Res. Inst. Publ., 33, Cambridge Univ. Press, Cambridge, 1998.

[21] Penrose, R. A generalized inverse for matrices. Proc. Cambridge Philos. Soc.51, (1955). 406-413.

[22] Porta, H.; Recht, L. Minimality of geodesics in Grassmann manifolds. Proc. Amer. Math. Soc. 100 (1987), no. 3, 464-466.

[23] Radjavi, H.; Williams, J. P. Products of self-adjoint operators. Michigan Math. J. 161969 $177-185$.

[24] Schmidt, E. Zür Theorie der linearen und nichtlinearen Integralgleichungen. I Teil. Entwicklung willkurlichen Funktionen nach System vorgeschriebener, Math. Ann., 63 (1907), 161-174.

[25] Slepian, D.; Pollak, H. O. Prolate spheroidal wave functions, Fourier analysis and uncertainty. I. Bell System Tech. J. 40 (1961), 43-63. 
[26] Smith, M. The spectral theory of Toeplitz operators applied to approximation problems in Hilbert spaces. Constr. Approx. 22 (2005), no. 1, 47-65.

(Esteban Andruchow) Instituto de Ciencias, Universidad Nacional de Gral. Sarmiento, J.M. Gutierrez 1150, (1613) Los Polvorines, Argentina and Instituto Argentino de Matemática, 'Alberto P. Calderón', CONICET, Saavedra 15 3er. piso, (1083) Buenos Aires, Argentina. e-mail: eandruch@ungs.edu.ar (Gustavo Corach) Instituto Argentino de Matemática, 'Alberto P. Calderón', CONICET, Saavedra 15 3er. piso, (1083) Buenos Aires, Argentina, and Depto. de Matemática, Facultad de Ingeniería, Universidad de Buenos Aires, Argentina.

e-mail: gcorach@fi.uba.ar 